\title{
The hypermultiplet with Heisenberg isometry in $N=2$ global and local supersymmetry
}

\author{
Nicola Ambrosetti, ${ }^{a}$ Ignatios Antoniadis, ${ }^{b, c}$ Jean-Pierre Derendinger ${ }^{a}$ and Pantelis \\ Tziveloglou ${ }^{b, d}$ \\ ${ }^{a}$ Albert Einstein Center for Fundamental Physics, Institute for Theoretical Physics, \\ Bern University, \\ Sidlerstrasse 5, CH-3012 Bern, Switzerland \\ ${ }^{b}$ Department of Physics, CERN — Theory Division, \\ CH-1211 Geneva, Switzerland \\ ${ }^{c}$ Centre de Physique Théorique, Ecole Polytechnique, \\ UMR du CNRS 7644, F-91128 Palaiseau, France \\ ${ }^{d}$ Department of Physics, Cornell University, \\ Ithaca, NY 14853, U.S.A. \\ E-mail: ambrose@itp.unibe.ch, ignatios.antoniadis@cern.ch, \\ derendinger@itp.unibe.ch, pt88@cornell.edu
}

ABSTRACT: The string coupling of $N=2$ supersymmetric compactifications of type II string theory on a Calabi-Yau manifold belongs to the so-called universal dilaton hypermultiplet, that has four real scalars living on a quaternion-Kähler manifold. Requiring Heisenberg symmetry, which is a maximal subgroup of perturbative isometries, reduces the possible manifolds to a one-parameter family that describes the tree-level effective action deformed by the only possible perturbative correction arising at one-loop level. A similar argument can be made at the level of global supersymmetry where the scalar manifold is hyper-Kähler. In this work, the connection between global and local supersymmetry is explicitly constructed, providing a non-trivial gravity decoupled limit of type II strings already in perturbation theory.

KEywords: Extended Supersymmetry, Superstrings and Heterotic Strings, Superstring Vacua

ARXIV EPRINT: 1005.0323 


\section{Contents}

1 Introduction 1

2 On the Heisenberg algebra and global supersymmetry 4

2.1 Lagrangians 4

$\begin{array}{lll}2.2 & \text { Hyper-Kähler metrics with Heisenberg symmetry } & 7\end{array}$

3 The universal hypermultiplet in $N=2$ supergravity $\quad 8$

3.1 The Calderbank-Pedersen metric with Heisenberg symmetry 8

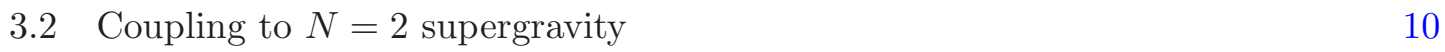

$\begin{array}{lll}3.3 & \mathrm{Sp}(2,4) & 11\end{array}$

3.4 The Heisenberg subalgebra of $\mathrm{SU}(1,2)$ and $\mathrm{Sp}(2,4) \quad 12$

$\begin{array}{ll}3.5 N=2 \text { supergravity scalar Lagrangian } & 13\end{array}$

$\begin{array}{ll}3.6 & \text { Solving the constraints } \\ \end{array}$

4 Zero-curvature hyper-Kähler limit $\quad 18$

$\begin{array}{llr}5 & \text { Conclusions } & 20\end{array}$

\section{Introduction}

Type II superstring theories compactified on a Calabi-Yau threefold $\left(\mathrm{CY}_{3}\right)$ yield $N=2$ supersymmetry in four dimensions. Even if gauge symmetries are absent in perturbation theory, the study of the effective field theory plays an important role in moduli stabilization by fluxes, as well as in a more realistic framework, such as in the presence of orientifolds and D-branes. Besides supergravity fields, their massless spectra involve vector multiplets and hypermultiplets describing all Kähler class and complex structure deformations of the $\mathrm{CY}_{3}$ manifold, as well as a universal hypermultiplet containing the string dilaton. Because of its special connection to the string coupling, the study of this universal hypermultiplet is an important problem per se. In the following, we restrict ourselves to this sector which also becomes exact in the particular case of a compactification with no Kähler class (complex structure) moduli in type IIB (IIA), or when these closed string moduli are fixed.

The dilaton hypermultiplet contains four real scalars parametrizing a quaternionKähler manifold, as required by $N=2$ supergravity [1]. Two of them come from the NS-NS (Neveu-Schwarz) sector and correspond to the string dilaton (associated to the string coupling constant) and the universal axion, Poincaré dual of the antisymmetric tensor $B_{\mu \nu}$. The other two come from the R-R (Ramond) sector and are obtained from various $n$-form gauge potentials. On the IIB side, they correspond to another scalar (0-form) $C_{0}$ and the dual of the two-form $C_{\mu \nu}$. At the string tree-level, these four scalars live on the 
symmetric coset $\mathrm{SU}(1,2) / \mathrm{SU}(2) \times \mathrm{U}(1)$ which is also Kähler [2-4]. Perturbative string corrections keep at least three isometries corresponding to the three independent shifts of the NS-NS axion and the R-R scalars, generating the Heisenberg algebra. Imposing just these isometries and the quaternion-Kähler structure, one finds that the only possible perturbative correction arises at one loop, destroying the Kähler structure of the manifold [5]. This correction was computed in $[5,6]$ and was found to be proportional to the Euler number of the $\mathrm{CY}_{3}$.

More precisely, in the context of IIB superstrings, the Heisenberg algebra is generated by a combination of the gauge symmetries of the two antisymmetric tensors $B_{\mu \nu}$ (NS-NS) and $C_{\mu \nu}(\mathrm{R}-\mathrm{R})$ and of the shift symmetry of the R-R scalar $C_{0}$ :

$$
\delta B_{\mu \nu}=2 \partial_{[\mu} \Lambda_{\nu]}, \quad \delta C_{\mu \nu}=2 \partial_{[\mu} \tilde{\Lambda}_{\nu]}+\lambda B_{\mu \nu}, \quad \delta C_{0}=\lambda .
$$

As a consequence, the theory depends on the invariant three-forms

$$
H_{\mu \nu \rho}=3 \partial_{[\mu} B_{\nu \rho]}, \quad F_{\mu \nu \rho}=3 \partial_{[\mu} C_{\nu \rho]}-C_{0} H_{\mu \nu \rho}
$$

and on $\partial_{\mu} C_{0}$. The Heisenberg algebra follows from

$$
\left[\delta_{1}, \delta_{2}\right] C_{\mu \nu}=2 \partial_{[\mu} \lambda_{2} \Lambda_{1 \nu]}-2 \partial_{[\mu} \lambda_{1} \Lambda_{2 \nu]} .
$$

After reduction to four dimensions, the gauge symmetries imply that each tensor can be dualized into a scalar field with axionic shift symmetry. The third global symmetry (with parameter $\lambda$ ) combines then with the axionic shifts to realize again the Heisenberg algebra on three scalar fields.

Indeed, one obtains three scalar fields $\varphi, \tau$ and $\eta=C_{0}$, with Heisenberg variations

$$
\delta \eta=c_{X}, \quad \delta \varphi=c_{Y}, \quad \delta \tau=c_{Z}-c_{X} \varphi .
$$

The scalars $\varphi$ and $\tau$ are Poincaré dual to $C_{\mu \nu}$ and $B_{\mu \nu}$, respectively. The duality relations are, schematically,

$$
\partial_{\mu} \varphi \sim \epsilon_{\mu \nu \lambda \rho} F^{\nu \lambda \rho}, \quad \partial_{\mu} \tau+\eta \partial_{\mu} \varphi \sim \epsilon_{\mu \nu \lambda \rho} H^{\nu \lambda \rho} .
$$

The algebra is $[X, Y] \sim Z$, with $Y$ and $Z$ generating the axionic shifts (with parameters $c_{Y}$ and $c_{Z}$ ), while $X$ generates the shift of the R-R scalar (with parameter $c_{X}$ ). Notice that the central charge of the algebra is (depending on the representation) the gauge symmetry of the R-R tensor and the axionic symmetry of $\tau$, dual to the NS-NS tensor.

Actually, as we will see later on, the Heisenberg algebra is extended by a fourth perturbative generator $M$ that rotates $X, Y$ and commutes also with the central charge $Z$ :

$$
\delta_{M} \eta=c_{M} \varphi, \quad \delta_{M} \varphi=-c_{M} \eta, \quad \delta_{M} \tau=\frac{c_{M}}{2}\left(\eta^{2}-\varphi^{2}\right) .
$$

Equivalently, $M$ rotates the phase of the complex R-R scalar $\eta+i \varphi$. As a result, the perturbative symmetry becomes the two-dimensional Euclidean group $E_{2}$ with central extension $Z$. 
Imposing $N=2$ supersymmetry and Heisenberg symmetry is a powerful constraint. In a previous work [7], we have briefly analyzed its implications in global $N=2$ supersymmetry, where hypermultiplet scalars form a Ricci-flat hyper-Kähler manifold [8]. For a single hypermultiplet, a unique non-trivial hyper-Kähler space admitting a one-parameter deformation is found. In local supersymmetry where hypermultiplets live on quaternionKähler manifolds [1], a one-parameter family of solutions emerges [5] from the general analysis of Calderbank and Pedersen [9]. These similar results suggest a correspondence between the local and global cases which could be studied using a Ricci-flat limit of the quaternion-Kähler manifold preserving the Heisenberg algebra. Our main goal is to analyze this correspondence using an explicit and intuitive construction of the supergravity couplings of the universal hypermultiplet

Taking the limit $\kappa \rightarrow 0$ in a hypermultiplet theory coupled to $N=2$ supergravity is a subtle problem. In contrast to the simplest case of $N=1, N=2$ supergravity imposes that the curvature of the hypermultiplet scalar manifold is proportional to the gravitational coupling $\kappa^{2}$ and hence the curved, quaternion-Kähler Einstein metric of the local hypermultiplet smoothly turns to a Ricci-flat hyper-Kähler metric. However, to obtain a non-trivial space, an appropriate limit must be defined, involving a new mass scale that should remain finite as the Planck mass goes to infinity. Some particular simple cases have been studied long ago, using the quaternionic quotient method [10,11], which however does not describe all quaternion-Kähler geometries for hypermultiplets coupled to $N=2$ supergravity. The construction associating hyperkähler cones (or rigid superconformal hypermultiplets) [12$15]$ and projective supergravity techniques $[16,17]$ is general, and a construction of the perturbative universal hypermultiplet has been obtained with this method [18]. In the following, we prefer to use a construction based on a quaternionic quotient which is simple and intuitive and displays clearly the algebraic structure underlying the theory.

Thus, in this work, we establish the precise connection between the local and global $N=2$ supersymmetric actions of a single hypermultiplet with Heisenberg isometry, to explicitly obtain the gravity-decoupled limit. We first reconstruct the $N=2$ supergravity Lagrangian using the method of quaternionic quotient, starting with superconformal supergravity and imposing gauge conditions and constraints. We then define the zero-curvature limit that reduces the perturbative-corrected metric of the dilaton hypermultiplet to the non-trivial hyper-Kähler form found in [7]. It turns out that the deformation parameter corresponding to the one-loop correction plays a crucial role. Indeed, the zero-curvature limit of the tree level $\mathrm{SU}(1,2) / \mathrm{SU}(2) \times \mathrm{U}(1)$ metric is trivial, leading to free kinetic terms. The presence of the one-loop parameter however allows for a non-trivial limit, giving rise to a hyper-Kähler metric that depends on a mass scale which remains finite as the fourdimensional Planck mass goes to infinity. At the same time, the value of the string coupling is tuned to a fixed value determined by the one-loop correction and can be made weak for large and positive Euler number of the $\mathrm{CY}_{3}$ manifold, so that non-perturbative corrections remain suppressed while taking the gravity decoupled limit.

This paper is organized as follows. In section 2, we recall the global construction of a four-dimensional hyper-Kähler manifold with the Heisenberg isometry in three formulations [7]: single-tensor which has the advantage of an off-shell $N=2$ supersymmetry 
formulation, scalar that provides a geometric description with a metric, and double tensor corresponding to the type IIB string basis. ${ }^{1}$ In section 3, we first review the CalderbankPedersen metric with Heisenberg symmetry and show that the latter is actually extended by a fourth generator which commutes with its central charge and rotates the other two. This generates a fourth perturbative isometry of the metric, as described above. We then rederive in supergravity the quaternion-Kähler metric with the Heisenberg isometry [5], by taking an appropriate quaternionic quotient of the symmetric quaternion-Kähler space $\mathrm{Sp}(2,4) / \mathrm{Sp}(2) \times \mathrm{Sp}(4)$ containing two hypermultiplets. Their reduction to one is achieved by gauging a symmetry corresponding to the central charge of the Heisenberg algebra. In section 4, we take the zero-curvature limit, leading to the one parameter hyper-Kähler manifold with Heisenberg symmetry of section 2. We thus find the correspondence of string fields in the rigid globally supersymmetric limit and we also discuss the coupling of the dilaton hypermultiplet to a D-brane where one of the two supersymmetries in non-linearly realized. Finally, section 5 contains some concluding remarks.

\section{On the Heisenberg algebra and global supersymmetry}

\subsection{Lagrangians}

Consider a $N=1$ globally supersymmetric theory with two superfields, a chiral $\Phi$ and a real linear $L$. It contains three real scalars, $\operatorname{Re} \phi=\left.\operatorname{Re} \Phi\right|_{\theta=0}, \operatorname{Im} \phi=\left.\operatorname{Im} \Phi\right|_{\theta=0}$, and $C=\left.L\right|_{\theta=0}$, and $L$ also depends on the curl of an antisymmetric tensor $H_{\mu \nu \rho}=3 \partial_{[\mu} B_{\nu \rho]}$. The Lagrangian (up to two derivatives) is

$$
\mathcal{L}=\int d^{2} \theta d^{2} \bar{\theta} \mathcal{H}(L, \Phi, \bar{\Phi})+\int d^{2} \theta W(\Phi)+\int d^{2} \bar{\theta} \bar{W}(\bar{\Phi})
$$

Besides the gauge invariance of $B_{\mu \nu}$ which does not act on the superfields, we also impose a two-parameter global symmetry acting on $\Phi$ with variations

$$
\delta \Phi=\alpha-i \beta
$$

In this formulation, all three symmetries trivially commute. Nevertheless, in the version where $B_{\mu \nu}$ is dualized to a scalar, or in the version where $\operatorname{Im} \phi$ (for instance) is transformed into a second antisymmetric tensor, the three-parameter symmetry realizes a Heisenberg algebra acting either on three scalars according to eq. (1.4), as in the hypermultiplet formulation of IIB strings compactified to four dimensions, or on two tensors and one scalar according to eqs. (1.1) and (1.3). The Lagrangian compatible with the required symmetry (2.2) has

$$
\mathcal{H}(L, \Phi, \bar{\Phi})=\mathcal{F}(L)+[A L+B] \Phi \bar{\Phi}, \quad W(\Phi)=k \Phi,
$$

with an arbitrary function $\mathcal{F}(L)$ and real constants $A$ and $B .^{2}$ The constant $k$ generates a $C$-dependent potential $V=|k|^{2} /(A C+B)$ which does not admit a vacuum if $A \neq 0$. We take then $k=0$.

\footnotetext{
${ }^{1}$ Note though that the basis of string vertex operators corresponds to the single-tensor representation [5].

${ }^{2}$ Of course, $B$ can be eliminated by a constant shift of $L$.
} 
The superfields $\Phi$ and $L$ provide an off-shell representation of the $N=2$ single-tensor multiplet. On the $N=1$ Lagrangian, the condition for a second supersymmetry is $[19]^{3}$

$$
\frac{\partial^{2} \mathcal{H}}{\partial L^{2}}+2 \frac{\partial^{2} \mathcal{H}}{\partial \Phi \partial \bar{\Phi}}=0
$$

which in turn indicates that

$$
\mathcal{F}_{N=2}(L)=-\frac{A}{3} L^{3}-B L^{2}
$$

The same theory is given by

$$
\widehat{\mathcal{F}}_{N=2}(L)=-\frac{1}{3 A^{2}}(A L+B)^{3} .
$$

Hence, the $N=2$ theory compatible with complex shift symmetry of $\Phi$ is the sum

$$
\mathcal{L}_{N=2}=\int d^{2} \theta d^{2} \bar{\theta}\left[A\left(-\frac{1}{3} L^{3}+L \Phi \bar{\Phi}\right)+B\left(-L^{2}+\Phi \bar{\Phi}\right)\right]
$$

of a trilinear interacting term and of a free term where the symmetry is trivial. If canonical dimensions are assigned to $L$ and $\Phi, A$ has dimension (mass) $)^{-1}$ and $B$ is dimensionless.

Fur further use, we need the bosonic component expansion of this superfield theory. Using

$$
\begin{aligned}
& L(x, \theta, \bar{\theta})=C+\theta \sigma^{\mu} \bar{\theta} v_{\mu}+\frac{1}{4} \theta \theta \overline{\theta \theta} \square C, \quad v_{\mu}=\frac{1}{6} \epsilon_{\mu \nu \rho \sigma} H^{\nu \rho \sigma}, \quad H_{\nu \rho \sigma}=3 \partial_{[\nu} B_{\rho \sigma]}, \\
& \Phi(x, \theta, \bar{\theta})=\phi(x)-i \theta \sigma^{\mu} \bar{\theta} \partial_{\mu} \phi-\theta \theta f-\frac{1}{4} \theta \theta \overline{\theta \theta} \square \phi,
\end{aligned}
$$

we obtain ${ }^{4}$

$$
\begin{aligned}
\mathcal{L}_{N=2, \text { bos. }}= & (A C+B)\left[\frac{1}{2}\left(\partial_{\mu} C\right)^{2}+\left(\partial_{\mu} \phi\right)\left(\partial^{\mu} \bar{\phi}\right)+\frac{1}{12} H^{\mu \nu \rho} H_{\mu \nu \rho}\right] \\
& -\frac{i}{12} A \epsilon^{\mu \nu \rho \sigma}\left(\bar{\phi} \partial_{\mu} \phi-\phi \partial_{\mu} \bar{\phi}\right) H_{\nu \rho \sigma} .
\end{aligned}
$$

Since, $\partial_{[\mu} H_{\nu \rho \sigma]}=0$, the variation (2.2) of $\phi$ induces a total derivative. Kinetic terms are positive if $A C+B>0$. If $A \neq 0, B$ can be eliminated by shifting $C$. The (shifted) field $C$ will be assumed strictly positive and the two options are an interacting, cubic theory with $A>0$ and $B=0$, or the free theory $A=0, B>0$.

We may then perform two supersymmetric duality transformations [20] on theory (2.3), either turning the linear $L$ into a chiral $S$ or turning the chiral $\Phi$ into a second linear multiplet $L^{\prime}$. The first transformation leads to

$$
\mathcal{L}=\int d^{2} \theta d^{2} \bar{\theta}[\widetilde{\mathcal{F}}(\mathcal{Y})+B \Phi \bar{\Phi}]
$$

where $\tilde{\mathcal{F}}(\mathcal{Y})$ is the Legendre transform of $\mathcal{F}(L)$ and the variable is ${ }^{5} \mathcal{Y}=S+\bar{S}+A \Phi \bar{\Phi}$. Invariance of $\mathcal{Y}$ under shift symmetries (2.2) requires a compensating variation of $S$ :

$$
\delta_{H} S=\left(\alpha \delta_{X}+\beta \delta_{Y}+\gamma \delta_{Z}\right) S=-A(\alpha+i \beta) \Phi+2 i \gamma,
$$

\footnotetext{
${ }^{3}$ The same conventions as in ref. [7] are used. They slightly differ from ref. [19].

${ }^{4}$ The auxiliary field $f$ vanishes.

${ }^{5}$ Notice that $\int d^{2} \theta d^{2} \bar{\theta} \Phi \bar{\Phi}=\frac{1}{A} \int d^{2} \theta d^{2} \bar{\theta} \mathcal{Y}+$ derivative.
} 
where the axionic shift symmetry of $\operatorname{Im} S$ is dual to the gauge symmetry of $B_{\mu \nu}$, and the subscripts $X, Y, Z$ make clear the correspondence with the transformations (1.4). Indeed, since

$$
\left[\delta_{H}^{\prime}, \delta_{H}\right] S \equiv-A\left(\alpha^{\prime}+i \beta^{\prime}\right) \delta_{H} \Phi+A(\alpha+i \beta) \delta_{H}^{\prime} \Phi=2 i A\left(\alpha^{\prime} \beta-\alpha \beta^{\prime}\right), \quad\left[\delta_{H}, \delta_{H}^{\prime}\right] \Phi=0,
$$

the chiral theory has Heisenberg symmetry. Moreover, the theory (2.9) has another symmetry $M$ rotating the chiral superfield $\Phi$, as already mentioned in the introduction (see eq. (1.5)).

For the $N=2$ single-tensor theory (2.7), the dual hypermultiplet theory ${ }^{6}$ is

$$
\mathcal{L}_{N=2}=\int d^{2} \theta d^{2} \bar{\theta} \mathcal{K}(\mathcal{Y})=\frac{2}{3 A^{2}} \int d^{2} \theta d^{2} \bar{\theta}\left(A \mathcal{Y}+B^{2}\right)^{3 / 2} .
$$

Eliminating some derivatives, the limiting case $A=0$ is a free theory. As required for a hyper-Kähler sigma-model, the determinant of the Kähler metric is constant (and positive).

A useful change of variable is

$$
\hat{S}=S-\frac{A}{2} \Phi^{2}, \quad \mathcal{Y}=\hat{S}+\overline{\hat{S}}+\frac{A}{2}(\Phi+\bar{\Phi})^{2} .
$$

and transformation (2.10) becomes $\delta_{H} \hat{S}=-2 A \alpha \Phi+2 i \gamma$. With these variables, the transformations with parameters $\beta$ and $\gamma$ only act as shift symmetries of $\operatorname{Im} \Phi$ and $\operatorname{Im} \hat{S}$ respectively. In terms of variables $\mathcal{Y}, \operatorname{Im} \hat{S}, \operatorname{Re} \Phi$ and $\operatorname{Im} \Phi$, one immediately deduces that the most general Heisenberg-invariant supersymmetric theory is of the form (2.9).

Performing the second duality transformation of the chiral $\Phi$ into a linear $L^{\prime}$, always leads to the dual theory

$$
\mathcal{L}=\int d^{2} \theta d^{2} \bar{\theta}\left[\mathcal{F}(L)-\frac{1}{2} \frac{L^{\prime 2}}{A L+B}\right],
$$

with $\mathcal{F}$ given in eq. (2.5). Expression (2.14) is actually the most general $N=1$ Lagrangian for $L$ and $L^{\prime}$ with symmetry

$$
\delta L^{\prime}=\alpha(A L+B)
$$

This transformation, which links the two antisymmetric tensors in $L$ and $L^{\prime}$ as in variation (1.1), forms with their respective gauge symmetries a Heisenberg algebra realized as in type IIB strings.

Instead of $\operatorname{Im} \Phi$, we could have chosen to dualize $e^{i a} \Phi$ for any phase $a$, since

$$
\int d^{2} \theta d^{2} \bar{\theta}(A L+B) \Phi \bar{\Phi}=\frac{1}{2} \int d^{2} \theta d^{2} \bar{\theta}(A L+B)\left(e^{i a} \Phi+e^{-i a} \bar{\Phi}\right)^{2}+\text { derivative. }
$$

The result would be again theory (2.14). This is a consequence of symmetry $M$, which is however fixed by the choice of dualization and does not act on $L^{\prime}$.

\footnotetext{
${ }^{6}$ With positive Kähler metric.
} 


\subsection{Hyper-Kähler metrics with Heisenberg symmetry}

The Kähler coordinates defined by $N=1$ chiral superfields $S$ and $\Phi$ are not necessarily the most appropriate to describe a hyper-Kähler manifold. There is a 'standard' set of coordinates used to describe hyper-Kähler metrics with shift isometries in the literature. For comparison purposes, we define in this subsection these coordinates in terms of our superfield components.

For any hyper-Kähler manifold with a shift symmetry, one can find coordinates in which the metric has the Gibbons-Hawking form [21, 22]

$$
d s^{2}=f(\vec{x}) d x_{i} d x_{i}+f(\vec{x})^{-1}\left(d \tau+\omega_{i} d x_{i}\right)^{2},
$$

with condition $\vec{\nabla} \times \vec{\omega}=\vec{\nabla} f$. Imposing the requirement of a Heisenberg symmetry acting according to

$$
\delta_{H} x_{1}=\sqrt{2} \alpha, \quad \delta_{H} x_{2}=-\sqrt{2} \beta, \quad \delta_{H} x_{3}=0, \quad \delta_{H} \tau=-\sqrt{2} \alpha x_{2}+\gamma
$$

also defines $d \tau+x_{1} d x_{2}$ as the invariant differential of $\tau$ and indicates that $\vec{\omega}=\left(0, x_{1}, 0\right)$. The value of $f(\vec{x})$ follows then from $\vec{\nabla} \times \vec{\omega}=\vec{\nabla} f$. This last condition is invariant under $\vec{\omega} \rightarrow \vec{\omega}+\vec{\nabla} \lambda(\vec{x})$, for any gauge function $\lambda(\vec{x})$. In turn, invariance of the metric requires the compensating transformation $\tau \rightarrow \tau-\lambda(\vec{x})$.

From the $N=2$ Kähler potential (2.12), the Kähler metric can be written ${ }^{7}$

$$
\begin{aligned}
d s^{2}= & \frac{1}{2}\left(A \mathcal{Y}+B^{2}\right)^{-1 / 2}\left[\frac{1}{4} d \mathcal{Y}^{2}+\left(d \operatorname{Im} S+i \frac{A}{2}(\Phi d \bar{\Phi}-\bar{\Phi} d \Phi)\right)^{2}\right] \\
& +\left(A \mathcal{Y}+B^{2}\right)^{1 / 2} d \Phi d \bar{\Phi}
\end{aligned}
$$

using coordinates $(\mathcal{Y}, \operatorname{Im} S, \operatorname{Re} \Phi, \operatorname{Im} \Phi)$. The supersymmetric duality transformation from $L$ to $S$ exchanges a real scalar $C=\left.L\right|_{\theta=0}$, invariant under Heisenberg variations, and $\operatorname{Re} S$ with variation (2.10). The Legendre transformation defines the change of variable from $\mathcal{Y}$ to $C$ :

$$
A C+B=\sqrt{A \mathcal{Y}+B^{2}} .
$$

Then, in terms of coordinates $(C, \operatorname{Im} S, \operatorname{Re} \Phi, \operatorname{Im} \Phi)$, the metric becomes

$$
d s^{2}=\frac{A C+B}{2}\left[d C^{2}+2 d \Phi d \Phi\right]+\frac{2}{(A C+B)}(d \tau+A \operatorname{Re} \Phi d \operatorname{Im} \Phi)^{2} .
$$

This is the Gibbons-Hawking metric $(2.16)$ with $\vec{x}=(\sqrt{2} \operatorname{Re} \Phi, \sqrt{2} \operatorname{Im} \Phi, C)$ and

$$
\tau=\frac{1}{2}(\operatorname{Im} S-A \operatorname{Re} \Phi \operatorname{Im} \Phi)=\frac{1}{2} \operatorname{Im} \hat{S} .
$$

The function

$$
f(\vec{x})=\frac{A C+B}{2}
$$

\footnotetext{
${ }^{7}$ From here on, we do not distinguish chiral superfields $S$ and $\Phi$ and their lowest complex scalar components.
} 
solves the hyper-Kähler condition $\vec{\nabla} \times \vec{\omega}=\vec{\nabla} f$ with $\vec{\omega}=\left(0, \frac{A}{2} x_{1}, 0\right)$. Choosing for instance $\lambda=-\frac{A}{2} x_{1} x_{2}$ turns then $\vec{\omega}$ into $\left(-\frac{A}{2} x_{2}, 0,0\right)$ and $d \tau+\frac{A}{2} x_{1} d x_{2}$ into $d \tau-\frac{A}{2} x_{2} d x_{1}$. Similarly, a rotation of $\Phi$

$$
\delta_{M} x_{1}=m x_{2}, \quad \delta_{M} x_{2}=-m x_{1},
$$

which is compatible with the shift symmetry $(2.2)$, corresponds to $\lambda(\vec{x})=\frac{A m}{4}\left(x_{2}^{2}-x_{1}^{2}\right)$. It is the isometry $M$ of metric (2.20).

The conclusion is that the Gibbons-Hawking Ansatz for the hyper-Kähler metric corresponds to coordinates where $\operatorname{Re} S$ is replaced by its Legendre dual $C$, which is also the lowest scalar component of the linear superfield dual to $S$.

\section{The universal hypermultiplet in $N=2$ supergravity}

Hypermultiplet scalars of $N=2$ supergravity live on $4 n$-dimensional quaternion-Kähler manifolds with holonomy included in $\operatorname{Sp}(2 n) \times \operatorname{Sp}(2)$. Supergravity requires that the curvature of these Einstein spaces is proportional to the gravitational coupling $\kappa^{2}$ [1]. Hence, the decoupling limit $\kappa \rightarrow 0$ turns the hypermultiplet manifold into a Ricci-flat hyper-Kähler space, as required by global $N=2$ supersymmetry [8]. For a single hypermultiplet, or a four-dimensional quaternion-Kähler manifold, the defining condition on the holonomy is not pertinent since $\mathrm{Sp}(2) \times \mathrm{Sp}(2) \sim \mathrm{SO}(4)$. The relevant condition is then self-duality of the Weyl tensor.

\subsection{The Calderbank-Pedersen metric with Heisenberg symmetry}

Calderbank and Pedersen [9] have classified all four-dimensional Einstein metrics with selfdual Weyl curvature and two commuting isometries. Using coordinates $(\rho, \eta, \varphi, \tau)$ with the isometries acting as shifts of $\varphi$ and $\tau$, their metrics are written in terms of any single function $F(\rho, \eta)$ verifying

$$
\frac{\partial^{2} F}{\partial \rho^{2}}+\frac{\partial^{2} F}{\partial \eta^{2}}=\frac{3 F}{4 \rho^{2}}
$$

It is simple to see [5] that metrics with Heisenberg symmetry are then obtained if $F$ does not depend on $\eta$, i.e. if ${ }^{8}$

$$
\sqrt{\rho} F(\rho)=\frac{1}{2}\left[\rho^{2}-\chi\right],
$$

with an arbitrary real parameter $\chi$. The Calderbank-Pedersen metric with Heisenberg symmetry (the $\mathrm{CPH}$ metric) reads then

$$
d s_{\mathrm{CPH}}^{2}=\frac{\rho^{2}+\chi}{\left(\rho^{2}-\chi\right)^{2}}\left(d \rho^{2}+d \eta^{2}+d \varphi^{2}\right)+\frac{4 \rho^{2}}{\left(\rho^{2}-\chi\right)^{2}\left(\rho^{2}+\chi\right)}(d \tau+\eta d \varphi)^{2} .
$$

The coordinate $\rho$ is positive, $\rho>0$, and positivity of the metric requires $\rho^{2}+\chi>$ 0 , a stronger condition if $\chi$ is negative. It is an Einstein metric with negative curvature, and it is Kähler only if $\chi=0$. Notice that if $\chi \neq 0$, the rescaling $(\rho, \eta, \varphi, \tau) \rightarrow$

\footnotetext{
${ }^{8}$ The metric does not make sense without the $\rho^{3 / 2}$ contribution to $F$ and the overall normalization of $F$ is a choice of coordinates. Our $\chi$ is $\hat{\chi}$ in ref. [5].
} 
$\left(|\chi|^{1 / 2} \rho,|\chi|^{1 / 2} \eta,|\chi|^{1 / 2} \varphi,|\chi| \tau\right)$ turns $\chi$ in metric (3.3) into \pm 1 . This is not true if we turn on string interactions, such as in the presence of D-branes where the dilaton, or equivalently the field $\rho$, couples to the Dirac-Born-Infeld (DBI) action in a non-trivial way (see section 4). For this reason, we keep explicitly $\chi$ throughout the paper. We may use a new coordinate $V=\rho^{2}$ with metric

$$
d s_{\mathrm{CPH}}^{2}=\frac{V+\chi}{(V-\chi)^{2}}\left(\frac{d V^{2}}{4 V}+d \eta^{2}+d \varphi^{2}\right)+\frac{4 V}{(V-\chi)^{2}(V+\chi)}(d \tau+\eta d \varphi)^{2} .
$$

The particular case $\chi=0$ has extended symmetry: it is the $\mathrm{SU}(2,1) / \mathrm{SU}(2) \times \mathrm{U}(1)$ metric with Kähler potential

$$
K(\hat{S}, \overline{\hat{S}}, \Phi, \bar{\Phi})=-\ln V, \quad V=\hat{S}+\overline{\hat{S}}-(\Phi+\bar{\Phi})^{2}
$$

and with $\Phi=\frac{1}{\sqrt{2}}(\eta+i \varphi), \tau=-\frac{1}{2} \operatorname{Im} \hat{S}$

The $\mathrm{CPH}$ metric is invariant under four isometry variations acting on coordinates $(\eta, \varphi, \tau):$

$$
\begin{aligned}
& \delta_{X} \eta=\sqrt{2}, \quad \delta_{Y} \eta=0, \quad \delta_{Z} \eta=0, \quad \delta_{M} \eta=\varphi, \\
& \delta_{X} \varphi=0, \quad \delta_{Y} \varphi=-\sqrt{2}, \quad \delta_{Z} \varphi=0, \quad \delta_{M} \varphi=-\eta, \\
& \delta_{X} \tau=-\sqrt{2} \varphi, \quad \delta_{Y} \tau=0, \quad \delta_{Z} \tau=1, \quad \delta_{M} \tau=\frac{1}{2}\left(\eta^{2}-\varphi^{2}\right) .
\end{aligned}
$$

The non-zero commutators are

$$
[X, Y]=2 Z, \quad[M, X]=Y, \quad[M, Y]=-X .
$$

Hence, $X, Y$ and $Z$ generate the Heisenberg algebra and $Z$ is a central extension of a twodimensional euclidean algebra generated by $M$ (which rotates $\varphi$ and $\eta$ ), $X$ and $Y$ (which translate $\varphi$ and $\eta$ ). With these conventions,

$$
\delta_{H} \Phi=(\alpha X+\beta Y+\gamma Z) \Phi=\alpha-i \beta, \quad \delta_{H} \hat{S}=4 \alpha \Phi-2 i \gamma
$$

and $V$ is invariant.

The metric (3.4) appears in the one-loop-corrected Lagrangian of the universal hypermultiplet of type II strings, reduced to four dimensions, with the NS-NS and R-R tensors dualized to scalars with shift symmetry [5]. At one-loop order, the four-dimensional dilaton field is related to coordinate $V$ and parameter $\chi$ by

$$
e^{-2 \phi_{4}}=V-\chi, \quad \chi=-\chi_{1}, \quad \chi_{1}=\frac{\chi_{E}}{12 \pi},
$$

where $\chi_{E}$ is the Euler number of the internal $\mathrm{CY}_{3}$ manifold. The real number $\chi_{1}$ encodes the one-loop correction [5]. Notice that this relation also indicates that $V-\chi=V+\chi_{1}>0$, which is stronger than $V=\rho^{2}>0$ if the Euler number is negative $(\chi>0)$. Since positivity of the $\mathrm{CPH}$ metric also requires $V+\chi>0$ if $\chi<0$, the domain of $V$ is naturally restricted to $V>|\chi|$. 
The R-R scalar is

$$
C_{0} \equiv \eta,
$$

and is shifted by symmetry $X$. Finally, Poincaré duality gives the following equivalences

$$
\begin{aligned}
d \varphi & \sim F_{3}=d C_{2}-\eta d B_{2}, \\
d \tau+\eta d \varphi & \sim H_{3}=d B_{2} .
\end{aligned}
$$

In the scalar version, the central charge is the shift $Z$ of $\tau$ (related to the NS-NS tensor $B_{2}$ ) while in the two-tensor version, it is the gauge variation of the (R-R) tensor $C_{2}$. Writing $\eta$ and $\varphi$ in a complex $\Phi$ is conventional: we always use

$$
\Phi=\frac{1}{\sqrt{2}}(\eta+i \varphi) .
$$

In the previous section, we found a unique four-dimensional hyper-Kähler manifold with Heisenberg symmetry. It also admits the fourth isometry $M$ rotating $\Phi$. In the quaternion-Kähler case, the theorem of Calderbank-Pedersen [9] leads then to a very similar uniqueness conclusion. We will see how these two results are connected when taking an appropriate zero-curvature limit. But we first want to obtain the $N=2$ supergravity coupling of the universal hypermultiplet on the $\mathrm{CPH}$ manifold.

\subsection{Coupling to $N=2$ supergravity}

As mentioned in the introduction, there are different methods to construct hypermultiplet couplings to $N=2$ supergravity. The simplest procedure, but not the most general, is to use hypermultiplets coupled to local $N=2$ superconformal symmetry $[23,24]$ and to perform a quaternionic quotient $[10,11]$ using supplementary hypermultiplet(s) and non-propagating vector multiplet(s). In this section, we use this procedure to obtain the supergravity theory of the one-loop-corrected dilaton hypermultiplet. Related constructions can be found in ref. [18], in the language of projective superspace or in ref. [25], using harmonic superspace.

Conformal $N=2$ supergravity is the gauge theory of $\mathrm{SU}(2,2 \mid 2)$, which has a $\mathrm{SU}(2)_{R} \times$ $\mathrm{U}(1)_{R} R$-symmetry with non-propagating gauge fields. Pure Poincaré $N=2$ supergravity is obtained from the superconformal coupling of one propagating vector multiplet ${ }^{9}$ (which may be charged under $\left.\mathrm{U}(1)_{R}\right)$ and one hypermultiplet (charged under $\left.\mathrm{SU}(2)_{R}\right)$ by gauge-fixing of the extraneous symmetries. These two multiplets include in particular the compensating fields used in the gauge-fixing to the Poincaré theory.

For the superconformal construction of our particular hypermultiplet sigma-model, we also need a physical hypermultiplet, with positive kinetic metric, to describe the dilaton multiplet. In addition, for the quaternionic quotient, we need a non-propagating vector multiplet with gauge field $W_{\mu}$, gauging a specific generator $T$ to be discussed below, and, since the elimination of the algebraic vector multiplet involves three constraints and one gauge choice on scalar fields, we also need a third non-physical hypermultiplet. Its kinetic

\footnotetext{
${ }^{9}$ Its gauge field is the graviphoton.
} 
metric can have a positive or negative sign, depending on the constraints induced by the choice of $T$. Hence, we need to consider the $N=2$ superconformal theory of two vector multiplets and three hypermultiplets. The superconformal hypermultiplet scalar sector has then an 'automatic' $\operatorname{Sp}(2,4)$ global symmetry in which the gauge generator $T$ of the quaternionic quotient is chosen.

\section{$3.3 \quad \mathrm{Sp}(2,4)$}

In the following, we consider three hypermultiplets coupled to (superconformal) $N=2$ supergravity. One (compensating) hypermultiplet has negative signature, the physical hypermultiplet has positive signature, the third hypermultiplet, associated to the nonpropagating vector multiplet, may have a positive or negative signature, depending on the constraints applied to the scalar fields. In any case, we are considering $\operatorname{Sp}(2,4)$-invariant supergravity couplings of $N=2$ hypermultiplets.

The hypermultiplet scalars are $A_{i}^{\alpha}$, with $\mathrm{SU}(2)_{R}$ index $i=1,2$ and $\operatorname{Sp}(2,4)$ index $\alpha=1, \ldots, 6$. They transform in representation $(\mathbf{6}, \mathbf{2})$ of $\mathrm{Sp}(2,4) \times \mathrm{SU}(2)_{R}$. Their conjugates $\operatorname{are}^{10}$

$$
A_{\alpha}^{i}=\left(A_{i}^{\alpha}\right)^{*}=\epsilon^{i j} \rho_{\alpha \beta} A_{j}^{\beta}
$$

with $\rho^{\alpha \beta} \rho_{\beta \gamma}=-\delta_{\gamma}^{\alpha}$ and $\epsilon^{i j} \epsilon_{j k}=-\delta_{k}^{i}$. We choose the $\operatorname{Sp}(2,4)$-invariant metric as

$$
\rho=I_{3} \otimes i \sigma_{2}=\left(\begin{array}{cc}
0 & I_{3} \\
-I_{3} & 0
\end{array}\right)
$$

and we use

$$
d=\left(\begin{array}{cc}
\eta & 0 \\
0 & \eta
\end{array}\right), \quad \eta=\operatorname{diag}(-1,1,-1), \quad \quad \rho d \rho=-d .
$$

In our choice of $\eta$, direction 1 corresponds to the superconformal compensator, direction 2 to the physical hypermultiplet and our choice of quaternionic quotient will require a negative metric in direction 3; otherwise, constraints cannot be solved. On scalar fields, $\operatorname{Sp}(2,4)$ acts according to

$$
\delta A_{i}^{\alpha}=g t^{\alpha}{ }_{\beta} A_{i}^{\beta}, \quad \delta A_{\alpha}^{i}=g t_{\alpha}{ }^{\beta} A_{\beta}^{i}, \quad t_{\alpha}{ }^{\beta}=-\rho_{\alpha \gamma} t^{\gamma} \rho^{\delta \beta} .
$$

Since relation (3.11) also implies $t_{\alpha}{ }^{\beta}=\left(t^{\alpha}{ }_{\beta}\right)^{*}$, the choice (3.12) and the invariance of $d_{\beta}^{\alpha} A_{\alpha}^{i} A_{i}^{\beta}$ lead to

$$
t=\left(\begin{array}{cc}
U & \eta Q \\
-\eta Q^{*} & U^{*}
\end{array}\right), \quad \quad U^{\dagger}=-\eta U \eta, \quad Q=Q^{\tau}, \quad t^{\dagger}=-d t d .
$$

This is an element of $\mathrm{Sp}(2,4): U$ generates the $\mathrm{U}(1,2)$ subgroup (9 generators) and $Q(12$ generators) generates $\operatorname{Sp}(2,4) / \mathrm{U}(1,2)$. The $(2 \times 2)$ matrix $A^{\dagger} d t A$, with matrix elements $A_{\alpha}^{i} d_{\beta}^{\alpha} t^{\beta}{ }_{\gamma} A_{j}^{\gamma}$, is antihermitian, as required by gauge invariance of $A^{\dagger} d A$, and traceless.

\footnotetext{
${ }^{10}$ We follow the conventions of ref. [24].
} 


\subsection{The Heisenberg subalgebra of $\mathrm{SU}(1,2)$ and $\mathrm{Sp}(2,4)$}

At string tree-level, the universal hypermultiplet of the dilaton in type II strings lives, when formulated in terms of four real scalars, on the quaternion-Kähler and Kähler manifold $\mathrm{SU}(1,2) / \mathrm{SU}(2) \times \mathrm{U}(1)=\mathrm{U}(1,2) / \mathrm{U}(2) \times \mathrm{U}(1)$. Since $\mathrm{U}(1,2)=\mathrm{SU}(1,2) \times \mathrm{U}(1)_{0}$ is maximal in $\mathrm{Sp}(2,4), \mathrm{Sp}(2,4)$ has a unique generator commuting with $\mathrm{SU}(1,2)$ : the generator of $\mathrm{U}(1)_{0}$. At one-loop however, the isometry is reduced and includes the Heisenberg algebra which is known to be a subalgebra of $\mathrm{SU}(1,2)$. We need to find the most general generator $T$ of $\operatorname{Sp}(2,4)$ which commutes with a Heisenberg subalgebra. In the following subsections, we will perform the quaternionic quotient construction induced by the gauging of $T$.

Since elements $U$ of the $\mathrm{U}(1,2)$ algebra verify $U^{\dagger}=-\eta U \eta$ and we have chosen $\eta=$ $\operatorname{diag}(-1,1,-1)$, a generic $U$ is

$$
U=\left(\begin{array}{ccc}
i a & A & B \\
\bar{A} & i b & C \\
-\bar{B} & \bar{C} & i c
\end{array}\right)
$$

with $a, b, c$ real, $A, B, C$ complex and elements of $\mathrm{SU}(1,2)$ are traceless. On a threedimensional complex vector, $\mathrm{U}(1,2)$ variations are $\delta A=U A$.

We may define the Heisenberg subalgebra as the $\mathrm{U}(1,2)$ transformations leaving $A_{1}-A_{2}$ invariant: $\left(\delta_{H} A\right)_{1}-\left(\delta_{H} A\right)_{1}=(U A)_{1}-(U A)_{2}=0$. The transformations acting on $A_{1}$ and $A_{2}$ are generated by the following three elements

$$
X=\left(\begin{array}{ccc}
0 & 0 & 1 \\
0 & 0 & 1 \\
-1 & 1 & 0
\end{array}\right), \quad Y=\left(\begin{array}{ccc}
0 & 0 & i \\
0 & 0 & i \\
i & -i & 0
\end{array}\right), \quad Z=\left(\begin{array}{ccc}
i & -i & 0 \\
i & -i & 0 \\
0 & 0 & 0
\end{array}\right)
$$

which verify

$$
0=X Z=Z X=Y Z=Z Y=Z^{2}, \quad X Y=-Y X=Z, \quad X^{2}=Y^{2}=i Z .
$$

The Heisenberg algebra

$$
[X, Y]=2 Z, \quad[X, Z]=[Y, Z]=0
$$

is then realized as a subalgebra of $\mathrm{SU}(1,2)$, with variations

$$
\delta_{H} A=(\alpha X+\beta Y+\gamma Z) A=\left(\begin{array}{ccc}
i \gamma & -i \gamma & \alpha+i \beta \\
i \gamma & -i \gamma & \alpha+i \beta \\
-\alpha+i \beta & \alpha-i \beta & 0
\end{array}\right)\left(\begin{array}{c}
A_{1} \\
A_{2} \\
A_{3}
\end{array}\right)
$$

in the fundamental representation. Since $Z$ is a central charge of the Heisenberg algebra, we are interested in the elements of $\mathrm{U}(1,2)$ which commute with $Z$. They form an algebra generated by five elements, $U_{0}, M, X, Y$ and $Z$, with

$$
U_{0}=i I_{3}, \quad M=i\left(\begin{array}{ccc}
1 & 0 & 0 \\
0 & 1 & 0 \\
0 & 0 & -2
\end{array}\right)
$$


$\left(U_{0}\right.$ generates the abelian factor of $\left.\mathrm{U}(1,2)=\mathrm{SU}(1,2) \times \mathrm{U}(1)_{0}\right)$. Besides the Heisenberg algebra generated by $X, Y, Z$, we also have

$$
[M, X]=3 Y, \quad[M, Y]=-3 X
$$

and $M$ generates a rotation of $(X, Y)$ leaving $X^{2}+Y^{2}=2 i Z$ invariant: $\left[M, X^{2}+Y^{2}\right]=$ $2 i[M, Z]=0$.

One then easily checks that the most general $\mathrm{U}(1,2)$ generator which commutes with the Heisenberg algebra generated by $X, Y, Z$ is proportional to

$$
\widehat{T}=U_{0}+\chi Z=i\left(\begin{array}{ccc}
1+\chi & -\chi & 0 \\
\chi & 1-\chi & 0 \\
0 & 0 & 1
\end{array}\right), \quad U_{0}=i I_{3}
$$

where $\chi$ is an arbitrary real number. If $\chi=0, \widehat{T}=U_{0}$ commutes with the whole $\mathrm{U}(1,2)$. If $\chi \neq 0, \widehat{T}$ commutes with the Heisenberg algebra supplemented by $U_{0}$ and $M$. The extension to $\operatorname{Sp}(2,4)$ is straightforward. Requiring that

$$
T=\left(\begin{array}{cc}
\hat{T} & 0 \\
0 & \hat{T}^{*}
\end{array}\right)
$$

in $\mathrm{Sp}(2,4)$ commutes with an element of $\mathrm{Sp}(2,4) / \mathrm{U}(1,2)$ corresponds to find a (nonzero) symmetric matrix $Q$ in eq. (3.15) such that $\hat{T}^{\dagger} Q$ is also antisymmetric, which is impossible. ${ }^{11}$ Hence, $T$ is also the most general generator in $\operatorname{Sp}(2,4)$ which commutes with the Heisenberg algebra generated by $X, Y$ and $Z$ in $\mathrm{SU}(1,2)$. It actually commutes with $X$, $Y, Z, M$ and $U_{0}$.

\section{5 $N=2$ supergravity scalar Lagrangian}

To construct the scalar kinetic metric, the relevant terms of the $N=2$ conformal supergravity Lagrangian are [10, 11, 23, 24]

$$
\begin{aligned}
e^{-1} \mathcal{L}= & d_{\beta}^{\alpha}\left(D_{\mu} A_{i}^{\beta}\right)\left(D^{\mu} A_{\alpha}^{i}\right)+\left(g d_{\beta}^{\alpha} A_{\alpha}^{i} T^{\beta}{ }_{\gamma} A_{k}^{\gamma} Y_{i}^{k}+\text { c.c. }\right) \\
& +\frac{1}{6} R\left(-X_{0} \bar{X}_{0}+d_{\beta}^{\alpha} A_{\alpha}^{i} A_{i}^{\beta}\right)+d\left(X_{0} \bar{X}_{0}+\frac{1}{2} d_{\beta}^{\alpha} A_{\alpha}^{i} A_{i}^{\beta}\right) .
\end{aligned}
$$

The complex scalar $X_{0}$ is the partner of the graviphoton, $Y_{j}^{i}, Y_{i}^{i}=0$, is the triplet of real auxiliary scalars in the non-propagating vector multiplet with gauge field $W_{\mu}$ used in the quaternionic quotient. The covariant derivatives are

$$
\begin{aligned}
& D_{\mu} A_{i}^{\alpha}=\partial_{\mu} A_{i}^{\alpha}-g^{\prime} W_{\mu} T^{\alpha}{ }_{\beta} A_{i}^{\beta}-g V_{\mu i}{ }^{j} A_{j}^{\alpha}, \\
& D_{\mu} A_{\alpha}^{i}=\partial_{\mu} A_{\alpha}^{i}-g^{\prime} W_{\mu} T_{\alpha}{ }^{\beta} A_{\beta}^{i}-g V_{\mu}{ }^{i}{ }_{j} A_{\alpha}^{j},
\end{aligned}
$$

\footnotetext{
${ }^{11}$ This would not be true for $\hat{T}=Z$, which commutes with a larger subalgebra of $\operatorname{Sp}(2,4)$. The $U_{0}$ component is necessary.
} 
where $g$ and $g^{\prime}$ are $\mathrm{SU}(2)_{R}$ and $\mathrm{U}(1)_{T}$ coupling constant. The (anti-hermitian) $\mathrm{SU}(2)$ gauge fields $V_{\mu i}{ }^{j}, V_{\mu i}{ }^{i}=0$, and the real auxiliary scalar $d$ belong to the multiplet of superconformal gauge fields:

$$
V_{\mu i}{ }^{j}=\frac{i}{2} V_{\mu}^{x}\left(\sigma^{x}\right)_{i}{ }^{j}, \quad V_{\mu}^{i}{ }_{j}=\epsilon^{i k} \epsilon_{j l} V_{\mu k}{ }^{l}=\left(V_{\mu i}{ }^{j}\right)^{*} .
$$

We will commonly use a matrix notation, with a $6 \times 2$ complex matrix $A$ and its $2 \times 6$ conjugate $A^{\dagger}$ replacing $A_{i}^{\alpha}$ and $A_{\alpha}^{i}$. Condition (3.11) implies that $A$ contains six complex components only. It also implies, in particular, that $A^{\dagger} d A=\frac{1}{2} \operatorname{Tr}\left(A^{\dagger} d A\right) I_{2}$. Since $V_{\mu}=$ $-V_{\mu}^{\dagger}$, the Lagrangian and the derivatives read

$$
\begin{aligned}
e^{-1} \mathcal{L}= & \operatorname{Tr}\left(D_{\mu} A^{\dagger}\right) d\left(D^{\mu} A\right)+g \operatorname{Tr} Y A^{\dagger} d T A+\text { c.c. } \\
& +\frac{1}{6} R\left(-X_{0} \bar{X}_{0}+\operatorname{Tr} A^{\dagger} d A\right)+d\left(X_{0} \bar{X}_{0}+\frac{1}{2} \operatorname{Tr} A^{\dagger} d A\right) ; \\
D_{\mu} A= & \partial_{\mu} A-g^{\prime} W_{\mu} T A-g A V_{\mu}, \\
D_{\mu} A^{\dagger}= & \partial_{\mu} A^{\dagger}-g^{\prime} W_{\mu} A^{\dagger} T^{\dagger}+g V_{\mu} A^{\dagger} .
\end{aligned}
$$

Constraints are obtained from the elimination of the auxiliary fields and from the gaugefixing of dilatation symmetry in the Poincaré theory:

- Einstein frame gauge-fixing condition and $d$ auxiliary field equation:

$$
X_{0} \bar{X}_{0}=\frac{1}{\kappa^{2}}, \quad \operatorname{Tr} A^{\dagger} d A=-\frac{2}{\kappa^{2}} .
$$

The second condition is invariant under $\mathrm{SU}(2)_{R}$ and $\mathrm{Sp}(4,2)$. With an $\mathrm{SU}(2)$ gauge choice, it allows to eliminate four scalar fields and would lead to the $\operatorname{Sp}(4,2) / \operatorname{Sp}(4) \times$ $\operatorname{Sp}(2)$ sigma-model.

- Auxiliary fields $Y_{j}^{i}$ :

$$
A^{\dagger} d T A=0 .
$$

Since this $2 \times 2$ matrix is traceless and antihermitian, these conditions eliminate three scalars and the associated abelian gauge invariance removes a fourth field.

The $\mathrm{SU}(2)_{R}$ gauge fields $V_{\mu i}{ }^{j}$ and the abelian $W_{\mu}$ have then algebraic field equations:

- Gauge field $W_{\mu}$, associated with generator $T$ :

$$
W_{\mu}=\frac{\operatorname{Tr}\left(\partial_{\mu} A^{\dagger} d T A-A^{\dagger} d T \partial_{\mu} A\right)}{2 g^{\prime} \operatorname{Tr}\left(A^{\dagger} T^{\dagger} d T A\right)} .
$$

- $\mathrm{SU}(2)_{R}$ gauge fields $V_{\mu i}{ }^{j}$ :

$$
V_{\mu}=-\frac{\partial_{\mu} A^{\dagger} d A-A^{\dagger} d \partial_{\mu} A}{g \operatorname{Tr}\left(A^{\dagger} d A\right)}
$$

According to the second eq. (3.28), the denominator is $-2 g / \kappa^{2}$. 
At this point, the scalar kinetic Lagrangian in theory (3.25) reduces to

$$
\begin{aligned}
e^{-1} \mathcal{L} & =e^{-1}\left(\mathcal{L}_{k i n .}+\mathcal{L}_{T}+\mathcal{L}_{\mathrm{SU}(2)}\right) \\
& =\operatorname{Tr}\left(\partial_{\mu} A^{\dagger}\right) d\left(\partial^{\mu} A\right)-g^{\prime 2} \operatorname{Tr}\left(A^{\dagger} T^{\dagger} d T A\right) W^{\mu} W_{\mu}-\frac{g^{2}}{\kappa^{2}} \operatorname{Tr}\left(V^{\mu} V_{\mu}\right) .
\end{aligned}
$$

The scalar fields are submitted to constraints (3.28) and (3.29) and the gauge fields $W_{\mu}$ and $V_{\mu i}{ }^{j}$ are defined by their field equations (3.30) and (3.31).

To study the constraints (3.28) and (3.29) for our specific choice (3.23) and (3.24) of gauged generator $T$, we introduce two three-component complex vectors:

$$
A_{i}^{\alpha}=\left(\begin{array}{cc}
\vec{A}_{+} & \vec{A}_{-} \\
-\vec{A}_{-}^{*} & \vec{A}_{+}^{*}
\end{array}\right), \quad \quad A_{\alpha}^{i}=\left(\begin{array}{cc}
\vec{A}_{+}^{*} & \vec{A}_{-}^{*} \\
-\vec{A}_{-} & \vec{A}_{+}
\end{array}\right),
$$

verifying the reality condition (3.11). On each doublet $A_{+a}, A_{-a}, a=1,2,3$, act two different $\mathrm{SU}(2)$ groups. Firstly, the superconformal $\mathrm{SU}(2)_{R}$ acts on \pm indices. Secondly, $\mathrm{Sp}(2,4) \supset \mathrm{Sp}(2)_{1} \times \mathrm{Sp}(2)_{2} \times \mathrm{Sp}(2)_{3} \sim \mathrm{SU}(2)_{1} \times \mathrm{SU}(2)_{2} \times \mathrm{SU}(2)_{3}$ and $\left(A_{+a},-A_{-a}^{*}\right)$ is a doublet of $\mathrm{SU}(2)_{a}$. One could define three quaternions

$$
Q_{a}=\left(\begin{array}{cc}
A_{+a} & A_{-a} \\
-A_{-a}^{*} & A_{+a}^{*}
\end{array}\right) \quad a=1,2,3
$$

with a left action of $\mathrm{SU}(2)_{a}$ and a right action of the superconformal $\mathrm{SU}(2)_{R}$. They verify (for each $a$ )

$$
Q_{a} Q_{a}^{\dagger}=Q_{a}^{\dagger} Q_{a}=\operatorname{det} Q_{a} I_{2}, \quad \operatorname{det} Q_{a}=\left|A_{+_{a}}\right|^{2}+\left|A_{-a}\right|^{2} .
$$

The second condition (3.28) from $N=2$ supergravity becomes:

$$
\vec{A}_{+}^{*} \cdot \vec{A}_{+}+\vec{A}_{-}^{*} \cdot \vec{A}_{-}=-\frac{1}{\kappa^{2}}, \quad \vec{A}^{*} \cdot \vec{A}=\vec{A}^{\dagger} \eta \vec{A}=-\left|A_{1}\right|^{2}+\left|A_{2}\right|^{2}-\left|A_{3}\right|^{2} .
$$

With eq. (3.24), condition (3.29) leads to three (real) equations:

$$
\begin{aligned}
& \vec{A}_{+}^{\dagger} i \eta \hat{T} \vec{A}_{+}=\vec{A}_{-}^{\dagger} i \eta \hat{T} \vec{A}_{-}, \\
& \vec{A}_{-}^{\dagger} i \eta \hat{T} \vec{A}_{+}=0
\end{aligned}
$$

$\left([i \eta \hat{T}]^{\dagger}=i \eta \hat{T}\right)$. With the explicit form of $\hat{T}$, eq. (3.23), and defining dimensionless fields $a_{ \pm i}=\sqrt{2} \kappa A_{ \pm i}$, the four constraints (3.36) and (3.37) read finally

$$
\begin{array}{lc}
I: & \left|a_{+1}\right|^{2}+\left|a_{-1}\right|^{2}-\left|a_{+2}\right|^{2}-\left|a_{-2}\right|^{2}+\left|a_{+3}\right|^{2}+\left|a_{-3}\right|^{2}=2, \\
I I: & -\left|a_{+1}\right|^{2}+\left|a_{+2}\right|^{2}-\left|a_{+3}\right|^{2}-\chi\left|a_{+1}-a_{+2}\right|^{2} \\
=-\left|a_{-1}\right|^{2}+\left|a_{-2}\right|^{2}-\left|a_{-3}\right|^{2}-\chi\left|a_{-1}-a_{-2}\right|^{2}, \\
\text { III : } \quad 0=-a_{+1} \bar{a}_{-1}+a_{+2} \bar{a}_{-2}-a_{+3} \bar{a}_{-3}-\chi\left(a_{+1}-a_{+2}\right)\left(\bar{a}_{-1}-\bar{a}_{-2}\right) .
\end{array}
$$

They are invariant under Heisenberg variations (3.20) of $\vec{a}_{+}$and $\vec{a}_{-}$. The case $\chi=0$ has been considered by Galicki [10]. Since it leads to $\mathrm{SU}(1,2) / \mathrm{SU}(2) \times \mathrm{U}(1)$, coordinates more appropriate for this larger isometry have been used. 


\subsection{Solving the constraints}

To solve the constraints (3.38), we insist on keeping in $\vec{a}_{-}$a field $\Phi$ which transforms under the Heisenberg variations ${ }^{12} \delta_{H} \vec{a}_{-}=(\alpha X+\beta Y+\gamma Z) \vec{a}_{-}$with a complex shift:

$$
\delta_{H} \Phi=\alpha-i \beta
$$

This is the case if $a_{-1}=a_{-2}$, and $a_{-3}$ is then invariant. We may define $\bar{\Phi}=a_{-1} / a_{-3}$ and constraint $I I I$ reduces to $a_{+3}=\left(a_{+2}-a_{+1}\right) \Phi$. Since

$$
\delta_{H}\left(\frac{a_{+2}+a_{+1}}{a_{+2}-a_{+1}}\right)=-2 i \gamma+2(\alpha+i \beta) \frac{a_{+3}}{a_{+2}-a_{+1}}=-2 i \gamma+2 \Phi \delta_{H} \bar{\Phi},
$$

we finally define

$$
S=\frac{a_{+2}+a_{+1}}{a_{+2}-a_{+1}}+Y, \quad \delta_{H} S=-2 i \gamma+2(\alpha+i \beta) \Phi
$$

and the quantity

$$
Y=S+\bar{S}-2 \Phi \bar{\Phi}
$$

is invariant under Heisenberg variations. The algebra follows from $\left[\delta_{H}^{\prime}, \delta_{H}\right]=\left(\alpha^{\prime} \beta-\right.$ $\left.\alpha \beta^{\prime}\right)[X, Y]=2\left(\alpha^{\prime} \beta-\alpha \beta^{\prime}\right) Z$ :

$$
\left[\delta_{H}^{\prime}, \delta_{H}\right] S=2\left(\alpha^{\prime}+i \beta^{\prime}\right) \delta_{H} \Phi-2(\alpha+i \beta) \delta_{H}^{\prime} \Phi=-4 i\left(\alpha^{\prime} \beta-\alpha \beta^{\prime}\right)=2\left(\alpha^{\prime} \beta-\alpha \beta^{\prime}\right) Z .
$$

These definitions are summarized in the choice

$$
\vec{a}_{-}=\frac{K}{\Delta}\left(\begin{array}{c}
\bar{\Phi} \\
\bar{\Phi} \\
1
\end{array}\right), \quad \vec{a}_{+}=\frac{1}{\Delta}\left(\begin{array}{c}
S-Y-1 \\
S-Y+1 \\
a
\end{array}\right)
$$

with complex fields $S, \Phi$ and $a$. The four available gauge choices have been used to take $\Delta=|\Delta|, K=|K|$ and $a_{-1}=a_{-2}$. Under Heisenberg variations, $\Delta$ and $K$ are invariant. Hence, we are left with eight real scalar fields submitted to the four constraints (3.38) which drastically simplify:

$$
\begin{array}{ll}
I: & \Delta^{2}\left(2-\left|a_{+1}\right|^{2}+\left|a_{+2}\right|^{2}-\left|a_{+3}\right|^{2}\right)=K^{2}, \\
I I: & 2(S+\bar{S})-|a|^{2}-4 Y=4 \chi-K^{2}, \\
\text { III : } & a=2 \Phi .
\end{array}
$$

Hence, the solution is

$$
\vec{a}_{-}=\sqrt{\frac{Y+2 \chi}{Y+\chi}}\left(\begin{array}{c}
\bar{\Phi} \\
\Phi \\
1
\end{array}\right), \quad \vec{a}_{+}=\frac{1}{\sqrt{2(Y+\chi)}}\left(\begin{array}{c}
S-Y-1 \\
S-Y+1 \\
2 \Phi
\end{array}\right) .
$$

\footnotetext{
${ }^{12}$ See eq. (3.20).
} 
The solution implies $Y+\chi>0$ if $\chi>0$ or $Y+2 \chi>0$ if $\chi<0$. The scalar kinetic Lagrangian (3.32) obtained from this solution is ${ }^{13}$

$$
\begin{aligned}
\kappa^{2} \mathcal{L}= & \frac{(Y+3 \chi)}{4(Y+2 \chi)(Y+\chi)^{2}}\left(\partial_{\mu} Y\right)^{2}-\frac{2}{Y+\chi} \partial_{\mu} \Phi \partial^{\mu} \bar{\Phi} \\
& +\frac{1}{2(Y+\chi)(Y+3 \chi)}\left[\operatorname{Im}\left(\partial_{\mu} S-2 \bar{\Phi} \partial_{\mu} \Phi\right)\right]^{2} \\
& +\frac{1}{2(Y+\chi)^{2}}\left[\operatorname{Im}\left(\partial_{\mu} S-2 \bar{\Phi} \partial_{\mu} \Phi\right)\right]^{2}+\frac{4(Y+2 \chi)}{(Y+\chi)^{2}} \partial_{\mu} \Phi \partial^{\mu} \bar{\Phi}
\end{aligned}
$$

The first line comes from the basic scalar kinetic terms $\mathcal{L}_{k i n}$. in Lagrangian (3.32). The second line is the contribution $\mathcal{L}_{T}$ of the gauge field of $T$, the third line arises from the supergravity $\mathrm{SU}(2)_{R}$ gauge fields. Each term is separately invariant under Heisenberg variations. Collecting terms, the final form of the theory is

$$
\begin{aligned}
\kappa^{2} \mathcal{L}= & \frac{Y+3 \chi}{(Y+\chi)^{2}}\left[\frac{1}{4} \frac{\left(\partial_{\mu} Y\right)^{2}}{Y+2 \chi}+2 \partial_{\mu} \Phi \partial^{\mu} \bar{\Phi}\right] \\
& +\frac{Y+2 \chi}{(Y+3 \chi)(Y+\chi)^{2}}\left(\partial_{\mu} \operatorname{Im} \hat{S}-4 \operatorname{Re} \Phi \partial_{\mu} \operatorname{Im} \Phi\right)^{2}
\end{aligned}
$$

where

$$
\hat{S}=S+\Phi^{2},
$$

for which $Y=\hat{S}+\overline{\hat{S}}-(\Phi+\bar{\Phi})^{2}$ and $\operatorname{Im}(d S-2 \bar{\Phi} d \Phi)=d \operatorname{Im} \hat{S}-4 \operatorname{Re} \Phi d \operatorname{Im} \Phi$. From the existence of solutions (3.44) and positivity of the Lagrangian, the range of $Y$ is $Y+\chi>0$ if $\chi>0$ and $Y+3 \chi>0$ if $\chi<0$. Writing as usual

$$
\mathcal{L}=\frac{1}{\kappa^{2}} g_{a b}\left(\partial_{\mu} q^{a}\right)\left(\partial^{\mu} q^{b}\right)=G_{a b}\left(\partial_{\mu} q^{a}\right)\left(\partial^{\mu} q^{b}\right),
$$

$q^{a}=(Y, \operatorname{Re} \Phi, \operatorname{Im} \Phi, \operatorname{Im} \hat{S})$, and comparing $d s^{2}=g_{a b} d q^{a} d q^{b}$ with expression (3.4), we see that the hypermultiplet kinetic metric $g_{a b}$ is the $\mathrm{CPH}$ metric with

$$
Y=V-2 \chi=\rho^{2}-2 \chi
$$

and with ${ }^{14}$

$$
\Phi=\frac{1}{\sqrt{2}}(\eta+i \varphi), \quad \operatorname{Im} \hat{S}=-2 \tau
$$

Positivity of kinetic terms is obtained if $V=\rho^{2}>|\chi|$ which is, as explained at the end of subsection 3.1, the natural domain of $V$.

As already observed, the case $\chi=0$ corresponds to the $\mathrm{SU}(2,1) / \mathrm{SU}(2) \times \mathrm{U}(1)$ metric

$$
d s^{2}=\frac{1}{Y^{2}}\left[\frac{1}{4} d Y^{2}+(d \operatorname{Im} \hat{S}-4 \operatorname{Re} \Phi d \operatorname{Im} \Phi)^{2}\right]+\frac{2}{Y} d \Phi d \bar{\Phi}
$$

\footnotetext{
${ }^{13}$ All fields and parameter $\chi$ are dimensionless.

${ }^{14}$ This choice is not unique. We may for instance rotate $\Phi$ using isometry $M$.
} 
With Kähler coordinates $\hat{S}$ and $\Phi$, the Kähler potential is $K=-\ln Y$, with $Y=V=$ $\hat{S}+\overline{\hat{S}}-(\Phi+\bar{\Phi})^{2}$.

This relatively simple construction of the one-loop-corrected dilaton hypermultiplet metric allows easily to derive the full $N=2$ supergravity Lagrangian, using $N=2$ superconformal tensor calculus.

\section{Zero-curvature hyper-Kähler limit}

All quaternion-Kähler metrics are Einstein spaces with nonzero curvature. With one hypermultiplet, the scalar kinetic Lagrangian (3.48) verifies [1]

$$
R_{a b}=-6 g_{a b}=-6 \kappa^{2} G_{a b}
$$

The link with global $N=2$ supersymmetry is realized by defining a $\kappa \rightarrow 0$ hyper-Kähler limit of the CPH metric (3.4) or (3.46) in which, if feasible, the Heisenberg algebra does not contract to an abelian symmetry. As observed in subsection 3.1, the magnitude of $\chi$ can be eliminated by rescaling of the coordinates (in the absence of D-branes). We then have three $|\chi|$-independent cases to examine: firstly, positive $\chi$, with $V>0$; secondly, $\chi=0$ $(V>0)$ which is $\mathrm{SU}(1,2) / \mathrm{SU}(2) \times \mathrm{U}(1)$; thirdly, a negative $\chi$, with $V>|\chi|$. In each case, we should seek to find a parameter-free zero-curvature limit. The most interesting case turns out to be $\chi$ negative, which we first study.

With $\chi$ negative, we are interested in the $\mathrm{CPH}$ metric in the region $V+\chi \sim 0$. We then apply to metric (3.4) the following change of variables:

$$
\begin{aligned}
V & =2|\chi| \kappa^{2 / 3} \mu^{-1 / 3} C-\chi, & \varphi & =\sqrt{|\chi|} \kappa^{2 / 3} \mu^{-1 / 3} \hat{\varphi}, \\
\eta & =\sqrt{|\chi|} \kappa^{2 / 3} \mu^{-1 / 3} \hat{\eta}, & \tau & =|\chi| \kappa^{4 / 3} \mu^{1 / 3} \hat{\tau},
\end{aligned}
$$

where $\mu$ is an arbitrary mass scale. Positivity of the metric, $V+\chi>0$ implies $C>0$. While the original fields are dimensionless, the new, hatted, fields $(C, \hat{\phi}, \hat{\eta}, \hat{\tau})$ have canonical dimension. With this choice of dependence in $\kappa$, the resulting metric is

$$
\begin{aligned}
d s^{2}=g_{a b} d q^{a} d q^{b}= & \frac{\kappa^{2}}{2} \frac{\mu C}{\left[(\kappa \mu)^{2 / 3} C+\mu\right]^{2}}\left[\frac{d C^{2}}{2 \kappa^{2 / 3} \mu^{-1 / 3} C+1}+d \hat{\eta}^{2}+d \hat{\varphi}^{2}\right] \\
& +\frac{\kappa^{2} \mu^{2}}{2 C} \frac{2(\kappa \mu)^{2 / 3} C+\mu}{\left[(\kappa \mu)^{2 / 3} C+\mu\right]^{2}}\left[d \hat{\tau}+\frac{1}{\mu} \hat{\eta} d \hat{\varphi}\right]^{2}
\end{aligned}
$$

since $\chi=-|\chi|$. Using this metric in Lagrangian (3.48), the overall factor $\kappa^{2}$ cancels and we can take the limit $\kappa \rightarrow 0$, with result

$$
\mathcal{L}_{\kappa \rightarrow 0}=\frac{C}{2 \mu}\left[\left(\partial_{\mu} C\right)^{2}+\left(\partial_{\mu} \hat{\eta}\right)^{2}+\left(\partial_{\mu} \hat{\varphi}\right)^{2}\right]+\frac{\mu}{2 C}\left[\partial_{\mu} \hat{\tau}+\frac{1}{\mu} \hat{\eta} \partial_{\mu} \hat{\varphi}\right]^{2} .
$$

This scalar Lagrangian has the hyper-Kähler metric with Heisenberg symmetry (2.20) with $A=1 / \mu$ and $B=0$ and with relations $\Phi=\frac{1}{\sqrt{2}}(\hat{\eta}+i \hat{\varphi}), \hat{\tau}=2 \tau$. As noticed earlier, parameter $B$ can always be absorbed in a shift of $C$, as long as $A \neq 0$. 
Notice that to obtain limit (4.4), we only need the change of variables (4.2) up to higher orders in $\kappa$. In particular, according to eq. (3.9), we may write the four-dimensional string dilaton as

$$
\begin{aligned}
e^{-2 \phi_{4}} & =2|\chi| \kappa^{2 / 3} \mu^{-1 / 3} C-2 \chi, \\
\phi_{4} & =\left\langle\phi_{4}\right\rangle-\kappa^{2 / 3} \mu^{-1 / 3} \hat{\phi}_{4}, \\
e^{-2\left\langle\phi_{4}\right\rangle} & =-2 \chi=2|\chi|, \quad C=2 \hat{\phi}_{4},
\end{aligned}
$$

in terms of the fluctuation $\hat{\phi}_{4}$ and of the background value $\left\langle\phi_{4}\right\rangle$. Since $|\chi|=\chi_{1}=\chi_{E} /(12 \pi)$, we are considering the case of a positive Euler number $\chi_{E}=2\left(h_{11}-h_{21}\right)$, with $h_{11}, h_{12}$ the corresponding Betti numbers of the $\mathrm{CY}_{3}$ manifold. A typical example with a single hypermultiplet would be IIA strings on a $\mathrm{CY}_{3}$ manifold with $h_{21}=0$. Positivity-related questions with several hypermultiplets, as is in particular the case with a negative Euler number, should be reanalyzed.

Comparing the scalings (4.2) and the identification of the string coupling in the last eq. (4.5), we see that the R-R fields $\eta$ and $\varphi$ carry as expected a supplementrary factor $g_{\text {string. }}$.

We could also consider the single-tensor version of the theory. Dualizing $\hat{\tau}$ into $H_{\mu \nu \rho}$, we find

$$
\begin{aligned}
\mathcal{L}_{\kappa \rightarrow 0, S T}= & \frac{C}{\mu}\left[\frac{1}{2}\left(\partial_{\mu} C\right)^{2}+\frac{1}{12} H^{\mu \nu \rho} H_{\mu \nu \rho}+\left(\partial_{\mu} \bar{\Phi}\right)\left(\partial^{\mu} \Phi\right)\right] \\
& -\frac{i}{12 \mu} \epsilon^{\mu \nu \rho \sigma}\left(\bar{\Phi} \partial_{\mu} \Phi-\Phi \partial_{\mu} \bar{\Phi}\right) H_{\nu \rho \sigma} .
\end{aligned}
$$

This is the bosonic sector (2.8) of the single-tensor theory (2.7) with again $A=1 / \mu$ and $B=0$. Then, for negative $\chi$, the $N=2$ supergravity hypermultiplet with Heisenberg symmetry is described in the global supersymmetry limit by the unique nontrivial theory with the same symmetry.

For completeness, we may also consider the case of the CPH metric with positive $\chi$. The interesting limiting regions are $V \sim 0$ and $V-\chi \sim 0$. If $V=\rho^{2} \ll \chi$,

$$
d s_{\mathrm{CPH}}^{2}=\frac{1}{\chi}\left(d \rho^{2}+d \eta^{2}+d \varphi^{2}\right)+\frac{4 \rho^{2}}{\chi^{3}}(d \tau+\eta d \varphi)^{2} .
$$

The appropriate rescalings are $(\rho, \eta, \varphi, \tau)=(\sqrt{\chi} \kappa \hat{\rho}, \sqrt{\chi} \kappa \hat{\eta}, \sqrt{\chi} \kappa \hat{\varphi}, \chi \hat{\tau})$ to obtain

$$
d s_{\mathrm{CPH}}^{2}=\kappa^{2}\left[d \hat{\rho}^{2}+d \hat{\eta}^{2}+d \hat{\varphi}^{2}+4 \hat{\rho}^{2}\left(d \hat{\tau}+\kappa^{2} \hat{\eta} d \hat{\varphi}\right)^{2}\right] .
$$

The Heisenberg symmetry acting on the rescaled fields has algebra $[X, Y]=2 \kappa^{2} Z$. In the limit $\kappa \rightarrow 0$, it contracts to $[X, Y]=0$ and we find

$$
\lim _{\kappa \rightarrow 0} \frac{1}{\kappa^{2}} d s_{\mathrm{CPH}}^{2}=d \hat{\rho}^{2}+4 \hat{\rho}^{2} d \hat{\tau}^{2}+d \hat{\eta}^{2}+d \hat{\varphi}^{2},
$$

which is the trivial four-dimensional euclidean space. The second region of interest if $\chi>0$ is $V-\chi \sim 0$. First, we change coordinates to

$$
V=2 \lambda C+\chi, \quad \eta=\lambda \hat{\eta} / \sqrt{\chi}, \quad \varphi=\lambda \hat{\varphi} / \sqrt{\chi}, \quad \tau=\lambda \hat{\tau}
$$


and the metric for $\lambda \rightarrow 0$ and $\chi$ finite reads

$$
d s_{\mathrm{CPH}}^{2}=\frac{1}{2 C^{2}}\left[d C^{2}+d \hat{\eta}^{2}+d \hat{\varphi}^{2}+d \hat{\tau}^{2}\right] .
$$

This limiting metric is $\mathrm{SO}(1,4) / \mathrm{SO}(4)$, again with $R_{i j}=-6 g_{i j}$ and with radius $\sim\langle C\rangle$. In the large radius, zero-curvature limit, the metric is trivial. Finally, in the $\mathrm{SU}(1,2) / \mathrm{SU}(2) \times$ $\mathrm{U}(1)$ case $\chi=0$, the zero-curvature limit is again trivial.

The conclusion is that in the zero-curvature limit, the CPH one-loop Lagrangian for the dilaton hypermultiplet is the hyper-Kähler $N=2$ sigma-model with Heisenberg symmetry (2.7). If the one-loop parameter $\chi$ is negative, then $A \neq 0$ and the Heisenberg algebra has a non-trivial realization in this limit. If $\chi \geq 0$ however, $A=0$ and the limit of $N=2$ global supersymmetry is the free hypermultiplet. In the string context, the above non-trivial limit can be taken if the string coupling is tuned at a fixed value, according to the third line of eq. (4.5), which applies with positive Euler number.

In ref. [7], we have constructed the interaction of a hypermultiplet with the Dirac-BornInfeld Maxwell Lagrangian. The hypermultiplet sector has a full linear $N=2$ supersymmetry while the second supersymmetry is nonlinearly realized on the Maxwell superfield $W_{\alpha}$. As an application of our results, we can easily use our identification of the string universal hypermultiplet. The bosonic DBI action, after elimination of the Maxwell auxiliary field and using the single-tensor formulation, is ${ }^{15}$

$$
\begin{aligned}
\mathcal{L}_{\mathrm{DBI}}= & \frac{1}{8 f}\left(2 g \operatorname{Re} \Phi-\frac{1}{f}\right)\left[1-\sqrt{1+\frac{2 g^{2} C^{2}}{\left(2 g \operatorname{Re} \Phi-\frac{1}{f}\right)^{2}}} \sqrt{-\operatorname{det}\left(\eta_{\mu \nu}+2 \sqrt{2} f F_{\mu \nu}\right)}\right] \\
& +g \epsilon^{\mu \nu \rho \sigma}\left(\frac{f}{4} \operatorname{Im} \Phi F_{\mu \nu} F_{\rho \sigma}-\frac{1}{4} B_{\mu \nu} F_{\rho \sigma}+\frac{1}{24 f} C_{\mu \nu \rho \sigma}\right) .
\end{aligned}
$$

In this expression, $f$ is the breaking scale of the second, nonlinearly realized supersymetry (with dimension (energy) ${ }^{-2}$ ) and $g$ is the Chern-Simons coupling ${ }^{16}$ (equal to the string coupling for a D3-brane). The four-form field $C_{\mu \nu \rho \sigma}$ is a component of the single-tensor multiplet required by supersymmetry of the nonlinear theory [7].

Since we have control of the kinetic Lagrangian of the universal string hypermultiplet in the global supersymmetry limit, we can then identify the single-tensor fields in terms of string fields. First, $C$ is the global dilaton and $B_{\mu \nu}$ is the NS-NS tensor. Then, the complex scalar $\Phi$ includes the R-R fields. The supersymmetric minimum of the scalar potential included in theory (4.12) implies $\langle C\rangle=0$ and $\Phi$ corresponds to flat directions of this vacuum.

\section{Conclusions}

In this work, we analyzed the effective field theory of the universal dilaton hypermultiplet of type II string compactifications on a $\mathrm{CY}_{3}$ manifold with a special emphasis on the global

\footnotetext{
${ }^{15}$ In ref. [7], this is the electric version of the theory, induced by a $N=2$ Chern-Simons coupling $g B \wedge F$.

${ }^{16}$ In contrast to ref. [7], we have defined single-tensor fields with canonical dimension so that $g$ has dimension (energy). We also chose the Fayet-Iliopoulos term to be $1 / \mathcal{F}$ so that gauge kinetic terms are canonically normalized at $\operatorname{Re} \Phi=0$.
} 
supersymmetry limit. The perturbative isometries form the two-dimensional Euclidean algebra $E_{2}$ with a central extension, which contains a Heisenberg subalgebra. Using this isometry as a guiding principle and the method of quaternionic quotient in conformal supergravity, we rederived the two-derivative $N=2$ supergravity action, depending on a deformation parameter that corresponds to the one-loop correction proportional to the Euler number of the $\mathrm{CY}_{3}$ manifold. We then established the precise connection with a one-parameter family of hyper-Kähler spaces in $N=2$ global supersymmetry, possessing the same isometry, by defining a non-trivial gravity decoupled limit characterized by a new mass scale. This requires the string coupling to be tuned at a fixed value which only occurs for a positive Euler number. As the latter becomes large, the theory becomes weakly coupled, justifying the perturbative approximation in taking the global limit. Notice that in the absence of moduli stabilization effects, this positivity requirement is compatible with the possibility of choosing $h_{21}=0$ in type IIA, that guarantees the absence of other hypermultiplets in the spectrum, which could modify the constraints we derived from the positivity of the metric. It would be interesting to understand this requirement in the general case.

\section{Acknowledgments}

This work has been supported by the Swiss National Science Foundation. The work of I.A. and P.T. was supported in part by the European Commission under the ERC Advanced Grant 226371 and the contract PITN-GA-2009-237920. I.A. was also supported by the CNRS grant GRC APIC PICS 3747. P.T. would like to thank the 'Propondis' Foundation for its support as well as the Galileo Galilei Institute for Theoretical Physics for the hospitality and the INFN for partial support during the early stage of this work.

Open Access. This article is distributed under the terms of the Creative Commons Attribution Noncommercial License which permits any noncommercial use, distribution, and reproduction in any medium, provided the original author(s) and source are credited.

\section{References}

[1] J. Bagger and E. Witten, Matter couplings in $N=2$ supergravity, Nucl. Phys. B 222 (1983) 1 [SPIRES].

[2] S. Cecotti, S. Ferrara and L. Girardello, Geometry of type II superstrings and the moduli of superconformal field theories, Int. J. Mod. Phys. A 4 (1989) 2475 [SPIRES].

[3] S. Cecotti, Homogeneous Kähler manifolds and T algebras in $N=2$ supergravity and superstrings, Commun. Math. Phys. 124 (1989) 23 [SPIRES].

[4] S. Ferrara and S. Sabharwal, Dimensional reduction of type II superstrings, Class. Quant. Grav. 6 (1989) L77 [SPIRES].

[5] I. Antoniadis, R. Minasian, S. Theisen and P. Vanhove, String loop corrections to the universal hypermultiplet, Class. Quant. Grav. 20 (2003) 5079 [hep-th/0307268] [SPIRES].

[6] I. Antoniadis, S. Ferrara, R. Minasian and K.S. Narain, $R^{4}$ couplings in $M$ - and type-II theories on Calabi-Yau spaces, Nucl. Phys. B 507 (1997) 571 [hep-th/9707013] [SPIRES]. 
[7] N. Ambrosetti, I. Antoniadis, J.P. Derendinger and P. Tziveloglou, Nonlinear supersymmetry, brane-bulk interactions and super-Higgs without gravity, Nucl. Phys. B 835 (2010) 75 [arXiv:0911.5212] [SPIRES].

[8] L. Álvarez-Gaumé and D.Z. Freedman, Geometrical structure and ultraviolet finiteness in the supersymmetric $\sigma$-model, Commun. Math. Phys. 80 (1981) 443 [SPIRES].

[9] D.M.J. Calderbank and H. Pedersen, Selfdual Einstein metrics with torus symmetry, J. Diff. Geom. 60 (2002) 485 [math/0105263].

[10] K. Galicki, Quaternionic Kähler and hyperkähler nonlinear $\sigma$-models, Nucl. Phys. B 271 (1986) 402 [SPIRES].

[11] K. Galicki, New matter couplings in $N=2$ supergravity, Nucl. Phys. B 289 (1987) 573 [SPIRES].

[12] B. de Wit, M. Roček and S. Vandoren, Hypermultiplets, hyperKähler cones and quaternion-Kähler geometry, JHEP 02 (2001) 039 [hep-th/0101161] [SPIRES].

[13] B. de Wit, B. Kleijn and S. Vandoren, Superconformal hypermultiplets, Nucl. Phys. B 568 (2000) 475 [hep-th/9909228] [SPIRES].

[14] A. Swann, Hyperkähler and quaternionic Kähler geometry, Math. Ann. 289 (1991) 421.

[15] K. Galicki, Geometry of the scalar couplings in $N=2$ supergravity models, Class. Quant. Grav. 9 (1992) 27 [SPIRES].

[16] A. Karlhede, U. Lindström and M. Roček, Selfinteracting tensor multiplets in $N=2$ superspace, Phys. Lett. B 147 (1984) 297 [SPIRES].

[17] S.J. Gates, Jr., C.M. Hull and M. Roček, Twisted multiplets and new supersymmetric nonlinear $\sigma$-models, Nucl. Phys. B 248 (1984) 157 [SPIRES].

[18] L. Anguelova, M. Roček and S. Vandoren, Quantum corrections to the universal hypermultiplet and superspace, Phys. Rev. D 70 (2004) 066001 [hep-th/0402132] [SPIRES].

[19] U. Lindström and M. Roček, Scalar tensor duality and $N=1, N=2$ nonlinear $\sigma$-models, Nucl. Phys. B 222 (1983) 285 [SPIRES].

[20] W. Siegel, Gauge spinor superfield as a scalar multiplet, Phys. Lett. B 85 (1979) 333 [SPIRES].

[21] S.W. Hawking, Gravitational instantons, Phys. Lett. A 60 (1977) 81 [SPIRES].

[22] G.W. Gibbons and S.W. Hawking, Gravitational multi-instantons, Phys. Lett. B 78 (1978) 430 [SPIRES].

[23] B. de Wit, J.W. van Holten and A. Van Proeyen, Transformation rules of $N=2$ supergravity multiplets, Nucl. Phys. B 167 (1980) 186 [SPIRES].

[24] B. de Wit, P.G. Lauwers and A. Van Proeyen, Lagrangians of $N=2$ supergravity-matter systems, Nucl. Phys. B 255 (1985) 569 [SPIRES].

[25] P.-Y. Casteill, E. Ivanov and G. Valent, $\mathrm{U}(1) \times \mathrm{U}(1)$ quaternionic metrics from harmonic superspace, Nucl. Phys. B 627 (2002) 403 [hep-th/0110280] [SPIRES]. 